\title{
Estudar, trabalhar ou alocar o tempo de outra maneira? Decisões dos jovens do sexo masculino no Brasil Metropolitano entre 2002 e $2015^{1}$
}

\author{
Anne Caroline Costa Resende \\ Universidade Federal de Minas Gerais \\ E-mail: anneccr@gmail.com \\ Mariangela Furlan Antigo \\ Universidade Federal de Minas Gerais \\ E-mail: maantigo@cedeplar.ufmg.br \\ Tomás de Faria Balbino \\ Universidade Federal de Minas Gerais \\ E-mail: tomasbalbino09@live.com \\ Carolina Guinesi Mattos Borges \\ Universidade Federal de Minas Gerais \\ E-mail:cgmb2@hotmail.com
}

Resumo: Este artigo busca estudar as decisões dos jovens de 18 a 24 anos do sexo masculino entre estudar, pertencer à pea ou alocar o tempo de outra maneira. A análise compreende o período entre 2002 a 2015, baseada nos dados da Pesquisa Mensal de Emprego do IBGE, quando esta tem sua série encerrada. Aplicada a seis regiões metropolitanas do país, a saber, Recife, Salvador, Belo Horizonte, Rio de Janeiro, São Paulo e Porto Alegre, os resultados apontam taxas de desocupação e de inatividade mais expressivas para jovens residentes nas regiões metropolitanas de Recife e de Salvador enquanto Porto Alegre figura com as menores taxas. Diferenças ainda mais evidentes ao se considerar a escolaridade dos jovens, com destaque para o grupo de 8 a 10 anos de estudo, no qual a taxa de inatividade da região metropolitana do Rio de Janeiro se destaca entre as mais altas. O emprego de matrizes de transição mostra um aumento na alocação do tempo em não estudar e não pertencer à população economicamente ativa (pea), com maior expressão para as regiões metropolitanas do Nordeste. Além disso, estimativas para cada região de um modelo logit multinomial, considerando a possibilidade de o jovem pertencer à pea e estar estudando, pertencer à pea e não estudar, não pertencer à pea e estudar e, ainda, não pertencer à pea e não estudar no período atual, mostram uma dependência temporal significativa da posição na qual ele se encontrava no ano anterior, evidenciando dificuldades para realizar transições, principalmente para aqueles que não se encontram na pea e não estudam. Jovens pretos e pardos, na condição de filhos, com menores níveis de escolaridade apresentam maior probabilidade de não pertencer à pea e não frequentar a escola. Fatores macroeconômicos contribuem para esse comportamento no período mais recente.

\footnotetext{
${ }^{1}$ Este estudo contou com o apoio financeiro da Fapemig (Edital 02/2016- Programa Pesquisador MineiroPPM X, Processo PPM-00658-16).
} 
Anne C. C. Resende; Mariangela F. Antigo; Tomás F. Balbino e Carolina G. Mattos Borges - p.50

Palavras-chave: jovens; alocação do tempo; nem-nem.

Abstract: This article aims to analyse the decisions of male youths between 15 and 24 years on allocating time either on studying, being in the labour market or through something else. Using data from the Monthly Employment Survey from IBGE for six metropolitan areas, Recife, Salvador, Belo Horizonte, Rio de Janeiro, São Paulo and Porto Alegre, the analysis comprehends the period between 2002 and 2016. The results have shown that unemployment rates and inactivity are more expressive for youths living in the metropolitan area of Salvador and Recife, meanwhile Porto Alegre has the smallest rates. The discrepancies are even more noticeable if youths' educational levels are considered, mostly with groups between 8 and 10 years of study, in which inactivity rates in the metropolitan area of Rio de Janeiro are amongst the highest. The use of the transition matrix have shown an upward trend of not allocating time for study and nor for being in the labour market, especially in the Northeast Metropolitan Areas. Furthermore, estimations using a multinomial logit model have been used for each metropolitan area considering four possibilities: studying and being in the labour market; not studying and being in the labour market; studying and not being the labour market; or not studying and not being in the labour market. In the recent period if the individual is not studying and nor is in the labour market, this is a sign of significant temporal dependency of how time was allocated in the last year, highlighting difficulties of mobility, especially for those who are not studying and nor in the labour market. Besides, young black men, in the status of being sons, with smaller levels of schooling presents higher probabilities of not studying and not being in the labour market. In recent years, macroeconomic factors also contribute for this behaviour.

Key-words: Yung people. Time allocation. NEET.

JEL Code: J11; J21; J24.

\section{INTRODUÇÃO}

A incidência de um grupo de jovens que não está participando da população economicamente ativa e não está estudando tem aumentado no período recente. Em termos produtivos, são jovens que não estão produzindo e não estão investindo em capital humano. Isso pode levar a uma redução na produtividade futura desses jovens, com reflexos negativos na economia (Borjas, 2012). Ver, também, Becker (1975). Ainda, um agravante pode ser pensado com relação à sua velhice. A falta de contribuição ao sistema previdenciário e a falta de investimento em capital humano, e, ainda, em capital humano absorvido pela inserção no mercado de trabalho, pode levar a comportamentos anti-sociais (Simmons, 2008). Os determinantes da população jovem nessa condição e no Brasil bem como suas características tem sido objeto de estudo de autores como Samoilenko e Carter (2015), Kovrova e Lion (2013), Camarano (2006), Cabanas, Komatsu 
e Menezes-Filho (2015), entre outros. Contudo, pouco se tem analisado sobre os determinantes da entrada nesta condição.

Nesse sentido, o presente artigo busca estudar as decisões dos jovens de 18 a 24 anos do sexo masculino entre estudar, pertencer à população economicamente ativa ou alocar o tempo de outra maneira. Para tanto, a análise compreende o período entre 2002 a 2015, baseada nos dados da Pesquisa Mensal de Emprego do IBGE, quando esta tem sua série encerrada. Empregando-se dois métodos de análise para as seis regiões metropolitanas do país abrangidas pela pesquisa, a saber, Recife, Salvador, Belo Horizonte, Rio de Janeiro, São Paulo e Porto Alegre, busca-se mensurar a influência de fatores individuais e relacionados à conjuntura sobre a decisão juvenil masculina de alocação de seu tempo.

\section{DEBATE BIBLIOGRÁFICO}

A alocação do tempo dos jovens entre fazer parte da população economicamente ativa, estudar ou combiná-lo de outra forma é referenciada no modelo de tomada de decisão juvenil sobre a alocação de tempo desenvolvido por Behrman et al. (2014). O modelo descreve como as restrições enfrentadas pelos jovens moldam suas decisões e de suas famílias no processo de acumulação de capital humano.

A Figura 1 fornece uma caracterização simples do processo de acumulação de capital humano antes e depois das chamadas "idades críticas". Os jovens alocam seu tempo entre quatro escolhas mutualmente excludentes, estudar (s), trabalhar (h), estudar e trabalhar (b) ou nenhum dos dois (n). Abaixo de a*, digamos aos 15 anos de idade, a criança é compelida a alocar seu tempo apenas para estudo devido às restrições legais para o trabalho. Acima da idade crítica $a^{*}$, os jovens decidem, dado o conjunto de restrições impostas por fatores microeconômicos, locais e, ainda, macroeconômicos, como alocar seu tempo entre: frequentar a escola, ir para o mercado de trabalho, fazer ambos ou nenhum dos dois. Ao atingir a idade crítica, a*, os jovens apresentam um conjunto de dotações imutáveis $\mathrm{Ha}^{*}$, que refletem fatores individuais e familiares, incluindo aspectos socioeconômicos e características demográficas; fatores macro e locais, como normas culturais e legais, disponibilidade e qualidade do ensino, entre outros. As dotações imutáveis incluem o tempo de escolaridade formal obtida até a idade $a^{*}$, habilidades cognitivas e habilidades socioemocionais. 
Figura 1: Acumulação de capital humano ao longo do ciclo de vida Capital Humano (Ha)

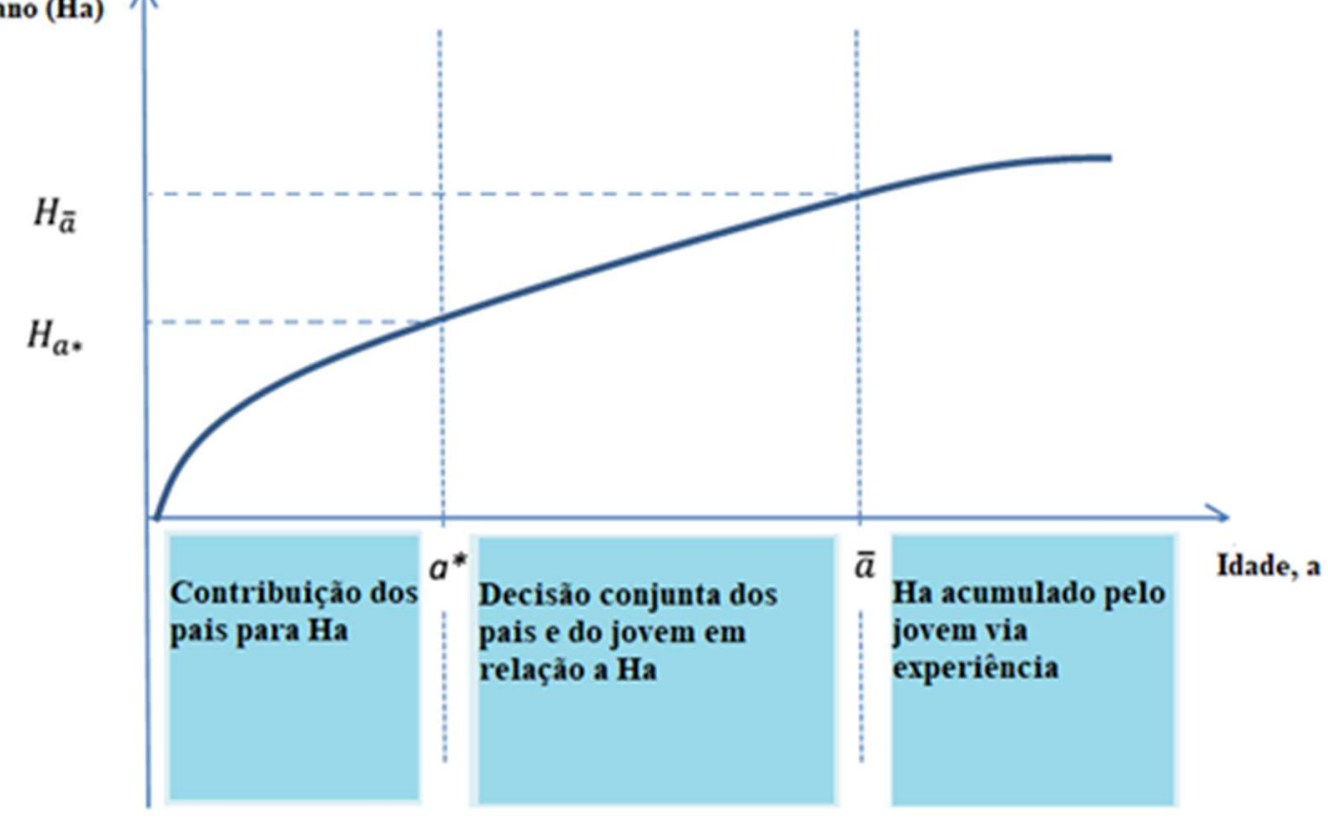

Fonte: Behrman et. Al (2014)

As dotações adquiridas ao atingir a idade a*, combinadas a experiência no mercado de trabalho e a educação formal determinam a acumulação de capital humano a partir de $a>a^{*}$. Um pressuposto do modelo é que a escola e o trabalho contribuem para o processo de acumulação de capital de humano, desta forma, os jovens que estão fora do sistema educacional e do mercado de trabalho tem esse processo interrompido. Entre as idades $a^{*}$ e $\bar{a}$, as decisões de acumulação de capital dos jovens são definidas em conjunto com seus pais. Acima da idade crítica à, digamos aos 24 anos de idade, o processo de acumulação de capital humano estará relacionado apenas aos anos de experiência no mercado de trabalho.

Evidências desse fenômeno tem sido objeto de estudo de diversos autores. Como exemplo, Cabanas, Komatsu e Menezes-Filho (2015) analisam a situação dos jovens de idade entre 15 e 24 anos entre 1992 a 2012. Com o emprego de um modelo logit multinomial e variáveis explicativas que envolvem aspectos do domicílio e características pessoais, os autores mostram que aumentos no nível de renda domiciliar reduzem o custo de oportunidade do estudo de jovens dependentes, favorecendo a dedicação aos estudos e desestimulando o emprego remunerado orientado a compor a renda familiar. Da mesma forma, aumentos na escolaridade dos pais aumentam a probabilidade do jovem estudar e reduzem a chance de que esteja trabalhando. Estes resultados apontam que 0 aumento recente da quantidade de jovens "nem-nem" ocorreu, dentre outros fatores, devido a aumentos reais na renda domiciliar das famílias brasileiras, uma vez que estes ganhos tendem a desestimular o emprego dos jovens e estimular menos que proporcionalmente a dedicação dos mesmos aos estudos.

Com o destaque para a duração da permanência de jovens na condição de "nemnem", Menezes-Filho, Cabanas e Komatsu (2013) por meio de matrizes de transição 
mostram redução, com o passar dos anos, na taxa de entrada na condição "nem-nem" para o subgrupo das mulheres, embora tanto homens como mulheres tenham experimentado aumentos significativos de duração média na condição. Também evidenciam que os jovens com ensino fundamental incompleto possuem tempo médio de permanência e taxa de entrada consideravelmente superiores aos demais grupos educacionais. A pesquisa sugere que o tempo médio de permanência na condição é relativamente pequeno, pois, conforme visto após um ano, a proporção dos jovens "nemnem" que trocam de situação é maior do que a proporção dos que permanecem sem estudar e sem trabalhar. Segundo os autores, aumentos da taxa de jovens nem-nem nos anos recentes correspondem ao aumento da duração média na condição, elevando o estoque de jovens que estão desocupados e que não estudam.

Piores resultados futuros em termos de escolaridade média, menor empregabilidade e maior incidência de inatividade para que quem permanece por muito tempo na condição de nem trabalhar e nem estudar são apresentados por Samoilenko e Carter (2015) No Brasil, os estudos sobre a taxa "nem-nem" tem se intensificado ao longo dos últimos anos, com uso, sobretudo, de microdados do Censo Demográfico e da Pesquisa Nacional por Amostra de Domicílios (PNAD). Camarano (2006) utiliza os dados dos censos demográficos de 1980 e 2000 para estimar modelos da probabilidade de se observar jovens entre 15 e 29 que nem trabalham e nem estudam, tomando como variáveis explicativas características domiciliares e demográficas, além da existência de deficiência física. Os resultados mostram que a educação tem o impacto de reduzir a probabilidade de ser "nem-nem" e que domicílios com pessoas nesta situação tendem a apresentar um menor nível de renda, além de contar principalmente com renda proveniente de programas sociais e doações. Além disso, observa importantes diferenças no comportamento dos jovens segundo o gênero. Vieira et. at (2016), utilizando dados da PNAD, de 1992 até 2013, estimam os efeitos de como mudanças no trabalho e na renda dos pais afetam as escolhas entre estudo e trabalho dos jovens. Os resultados indicam que o crescimento da renda da mãe tem um efeito maior no aumento da proporção de jovens que estudam, quando comparado ao crescimento da renda do pai. Os resultados mostram também que se ambos os pais trabalham, as probabilidades de 0 jovem ser "nem-nem" ou a de ele somente participar da PEA diminuem.

Em síntese, a literatura teórica e empírica mostra a importância de se considerar os aspectos tanto domiciliares como pessoais dos jovens em sua probabilidade de ser "nem-nem". Contudo, a maior parte da literatura tem dedicado espaço a discutir a determinação estática da taxa "nem-nem" no país, à exceção do estudo de MenezesFilho, Cabanas e Komatsu (2013) que aborda a saída desta situação em uma análise dinâmica.

\section{BASE DE DADOS E MÉTODOS DE ANÁLISE}

Para a análise da decisão de alocação do tempo dos jovens do sexo masculino de 18 a 24 anos, utiliza-se a Pesquisa Mensal de Emprego (PME), realizada pelo Instituto Brasileiro de Geografia e Estatística (IBGE), durante o período de 2002 a 2015. O levantamento amostral é feito para seis regiões metropolitanas do Brasil, a saber, Recife, 
Salvador, Belo Horizonte, Rio de Janeiro, São Paulo e Porto Alegre e tem a vantagem de acompanhar o indivíduo no intervalo de até um ano.

A influência de fatores individuais e relacionados à conjuntura sobre a decisão juvenil de estudar, trabalhar ou alocar o tempo de outra maneira é feita por uma análise univariada por matrizes de transição e, uma multivariada, com o emprego de um modelo logit multinomial. O primeiro método se baseia no trabalho de Clark e Summers (1990), em que o comportamento do jovem, segundo suas características individuais, pode ser representado por uma matriz de probabilidade de transição, ou matriz de Markov. A alocação do tempo juvenil é dada pela decisão entre fazer parte da pea e estudar ou não estudar e, ainda, não fazer parte da pea e estudar ou não estudar. É ser representada pela probabilidade de o jovem $i$ estar no estado $k$ no período $t+1$, condicionado ao fato de ele se encontrar no estado / no período $t$. Trata-se as transições entre os estados como um processo de Markov, no qual um estado estacionário é alcançado, independente de condições iniciais e a proporção de estado estacionário em cada estado é encontrada como uma função da matriz de transição inteira.

A segunda metodologia adotada baseia-se em uma análise econométrica multivariada, na qual estima-se um modelo Logit Multinomial presente em Wooldridge (2007). Com isso, é possível entender como as mudanças no conjunto de variáveis relativas a atributos pessoais e à conjuntura macroeconômica afetam a probabilidade de um jovem pertencer à pea e estudar, pertencer à pea e não estudar, não pertencer à pea e estudar ou, ainda, não pertencer à pea e não estudar no momento da quinta entrevista. As variáveis explicativas abarcadas pelo modelo são: categoria no período da primeira entrevista: pertence à pea e estuda, pertence à pea e não estuda, não pertence à pea e estuda, não pertence à pea e não estuda; cor; condição no domicílio; anos de estudo; idade; e, ainda, dummies anuais de 2003 a 2015. Os modelos foram estimados separadamente para cada região metropolitana contemplada na pesquisa.

\section{DISCUSSÃO DOS RESULTADOS}

Os resultados apresentados no Gráfico 1 mostram uma tendência crescente a partir de 2013 para a taxa de desocupação juvenil masculina nas regiões metropolitanas das principais capitais do Brasil. As regiões metropolitanas de Salvador e de Recife se destacam com as maiores taxas entre 2003 e 2015 enquanto a região metropolitana de Porto Alegre apresenta a mais baixa, seguida pela região metropolitana de Belo Horizonte. 
p. 55 - Estudar, trabalhar ou alocar o tempo de outra maneira?

Gráfico 1- Taxa de desocupação para jovens do sexo masculino, por região metropolitana, 2002- 2015

Desocupação

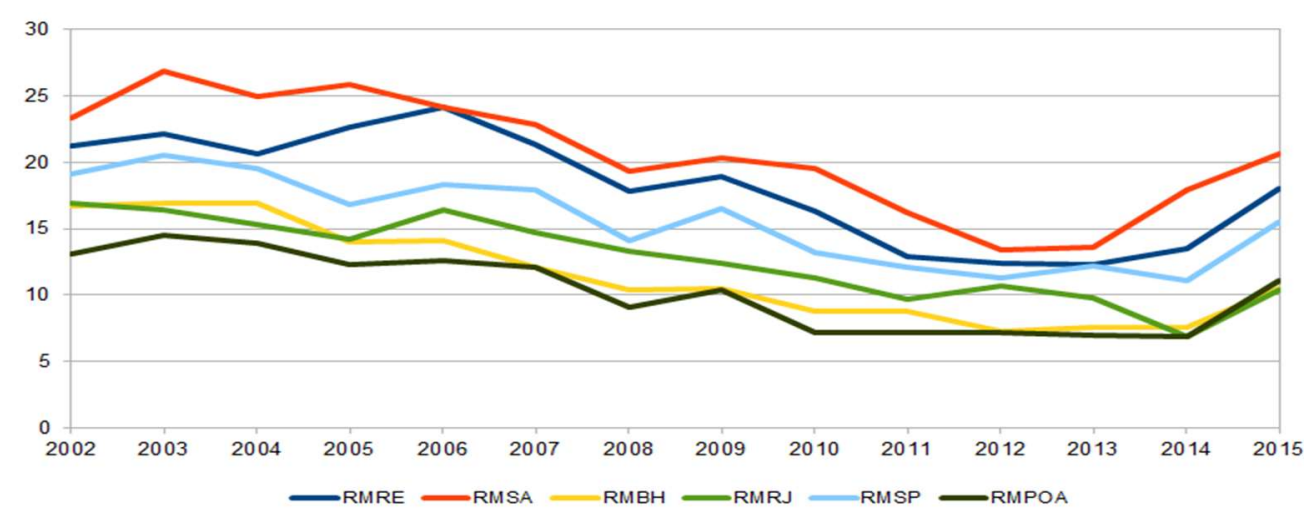

Fonte: Elaboração própria a partir dos microdados da PME.

A taxa de inatividade para cada região metropolitana pode ser vista no gráfico 2 . Pela leitura do gráfico abaixo, apreende-se um comportamento levemente crescente da taxa para as regiões, mas sem grandes pontos de inflexão. O que se observa é que a região metropolitana do Rio de Janeiro se integra às regiões metropolitanas de Salvador e Recife com as piores taxas de inatividade. Para a taxa de desocupação, a região metropolitana do Rio figurava entre as menores taxas. Cabe destacar também os menores números registrados pela região metropolitana de Porto Alegre acompanhados das menores taxas de desocupação. Tanto a taxa de desocupação quanto a de inatividade mostram comportamentos distintos regionalmente. Enquanto as capitais do nordeste se destacam pelas maiores taxas, as capitais do Sul e Sudeste apresentam os menores valores.

Gráfico 2- Taxa de inatividade para jovens do sexo masculino, por região metropolitana, 2002-2015

Taxa de inatividade

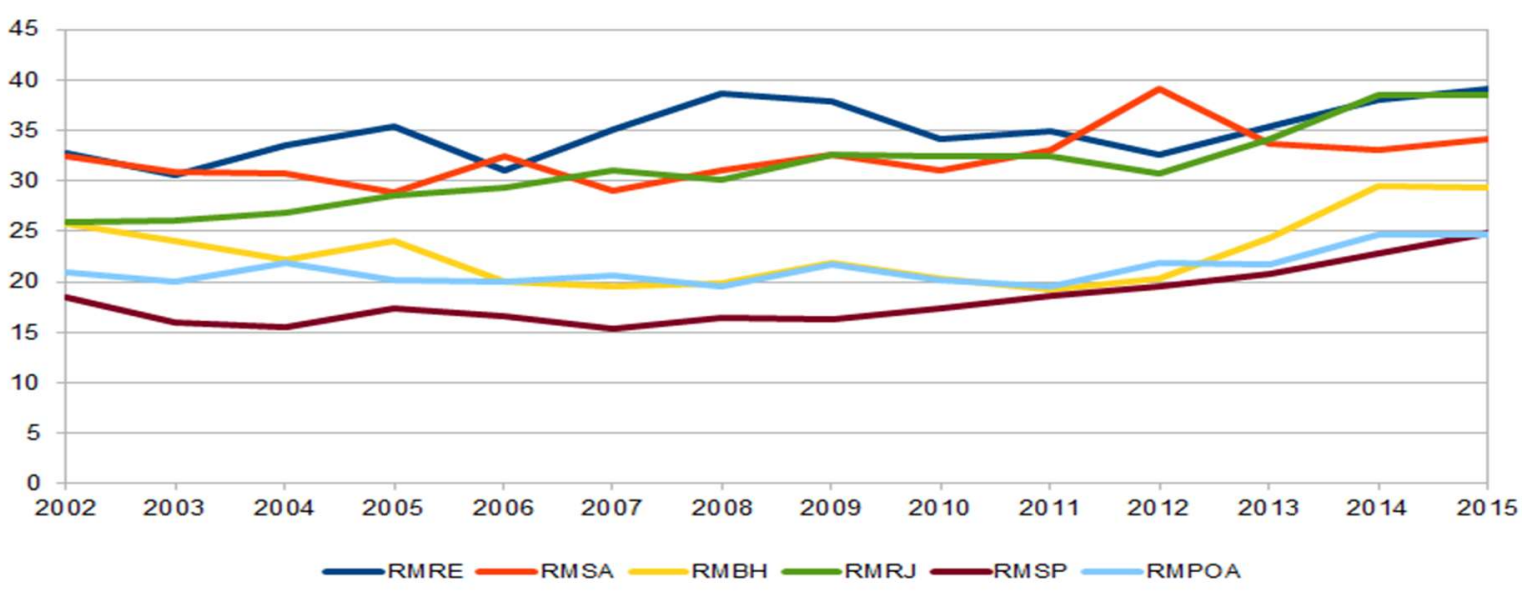

Fonte: Elaboração própria a partir dos microdados da PME 
Ao analisar as taxas de desocupação por grupos de escolaridade (Gráfico 3), o comportamento temporal se mantém próximo ao observado na análise das taxas totais, com as regiões de Salvador e de Recife mantendo taxas superiores ao restante.

Gráfico 3- Taxa de desocupação para jovens do sexo masculino, por região metropolitana e escolaridade, 2002-2015

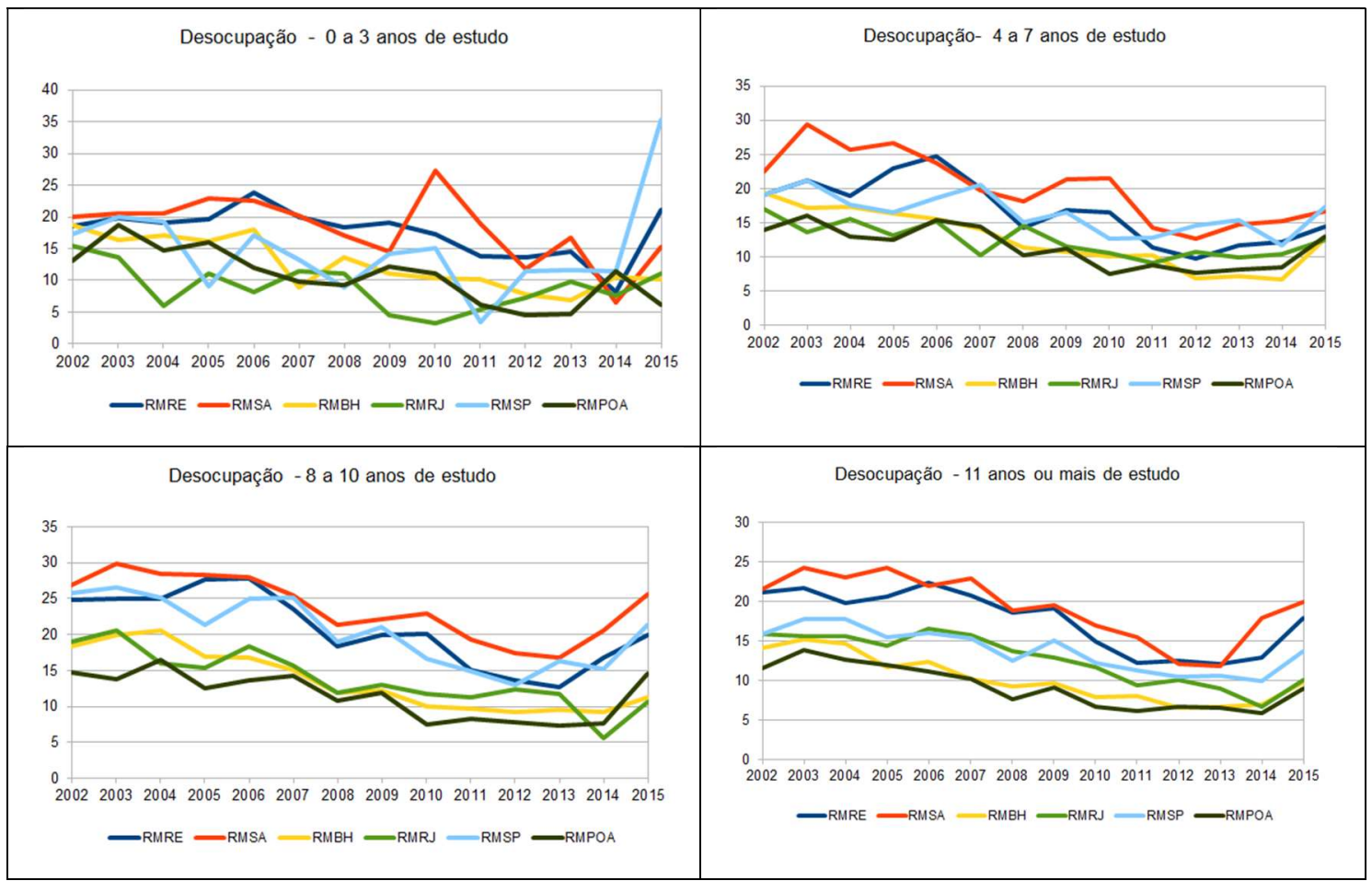

Fonte: Elaboração própria a partir dos microdados da PME

A taxa de inatividade por grupos de escolaridade pode ser visualizada no Gráfico 4. Com tendência de aumento entre 2002 e 2015, tem-se um menor efeito regional para jovens de menor escolaridade ( 0 e 3 anos de estudo). Para jovens com escolaridade entre 4 e 7 anos, a taxa de inatividade passa a ser mais expressiva nas regiões metropolitanas de Recife e Salvador. Para aqueles com maior escolaridade essa tendência é acompanhada, também, pelos jovens residentes na região metropolitana do Rio de Janeiro. 
Gráfico 4- Taxa de inatividade para jovens do sexo masculino, por região metropolitana e escolaridade, 2002-2015

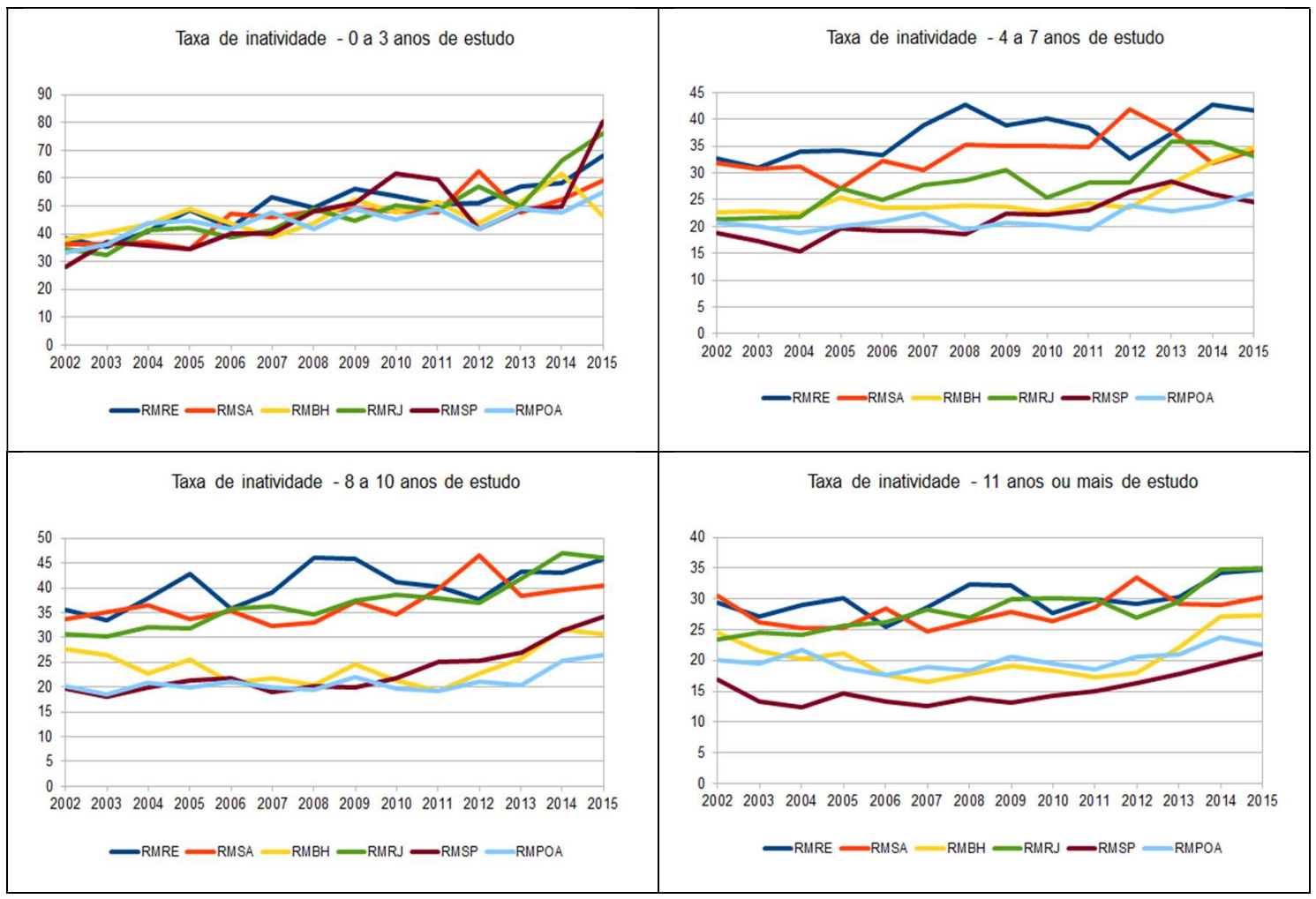

Fonte: Elaboração própria a partir dos microdados da PME

Tendo em vista a diferença de participação juvenil do sexo masculino no mercado de trabalho, ainda mais expressiva ao se considerar a escolaridade, busca-se compreender a decisão do jovem de como alocar o seu tempo. Nesse sentido, quatro grupos são considerados: aqueles que optam por fazer parte da pea e estudar, aqueles que optam por fazer parte da pea e não estudar, aqueles que optam por não fazer parte da pea e estudar e, ainda, aqueles que optam por não fazer parte da pea e não estudar.

Considerando a primeira e a quinta entrevista, bem como a probabilidade de transitar ou não entre as categorias, estima-se a fração de tempo que o jovem alocaria para cada uma, a partir de suas probabilidades. Os resultados podem ser vistos no gráfico abaixo. Pela sua leitura tem-se que o tempo alocado para fazer parte da pea e estudar tem adquirido uma tendência crescente nos últimos anos. Ainda, fazer parte da pea e não estudar apresenta comportamento constante em torno de uma média durante todo o período analisado em todas regiões metropolitanas.

Contudo, cabe destacar a parcela do tempo para não fazer parte da pea e não estudar, que apresenta um comportamento negativo, sobretudo, para Salvador. A alocação do tempo em não fazer parte da pea e não estudar também seguiu uma leve tendência de crescimento nos anos observados. A região metropolitana de Recife apresenta os piores números dentro desse grupo acompanhada de Salvador.

Esses resultados merecem destaque pois jovens que não fazem parte da pea e não estudam deixam de acumular capital humano em uma fase importante do ciclo de vida. Estudar pode ser considerada uma decisão de investimento de um período mais longo do 
tempo, que irá gerar resultados futuros sobre sua ocupação e sua renda. Essa condição pode comprometer sua capacidade produtiva em um futuro próximo.

Gráfico 5-Percentual do tempo alocado pelos jovens do sexo masculino para fazer (ou não) parte da pea e estudar (ou não), por região metropolitana, 2002-2015

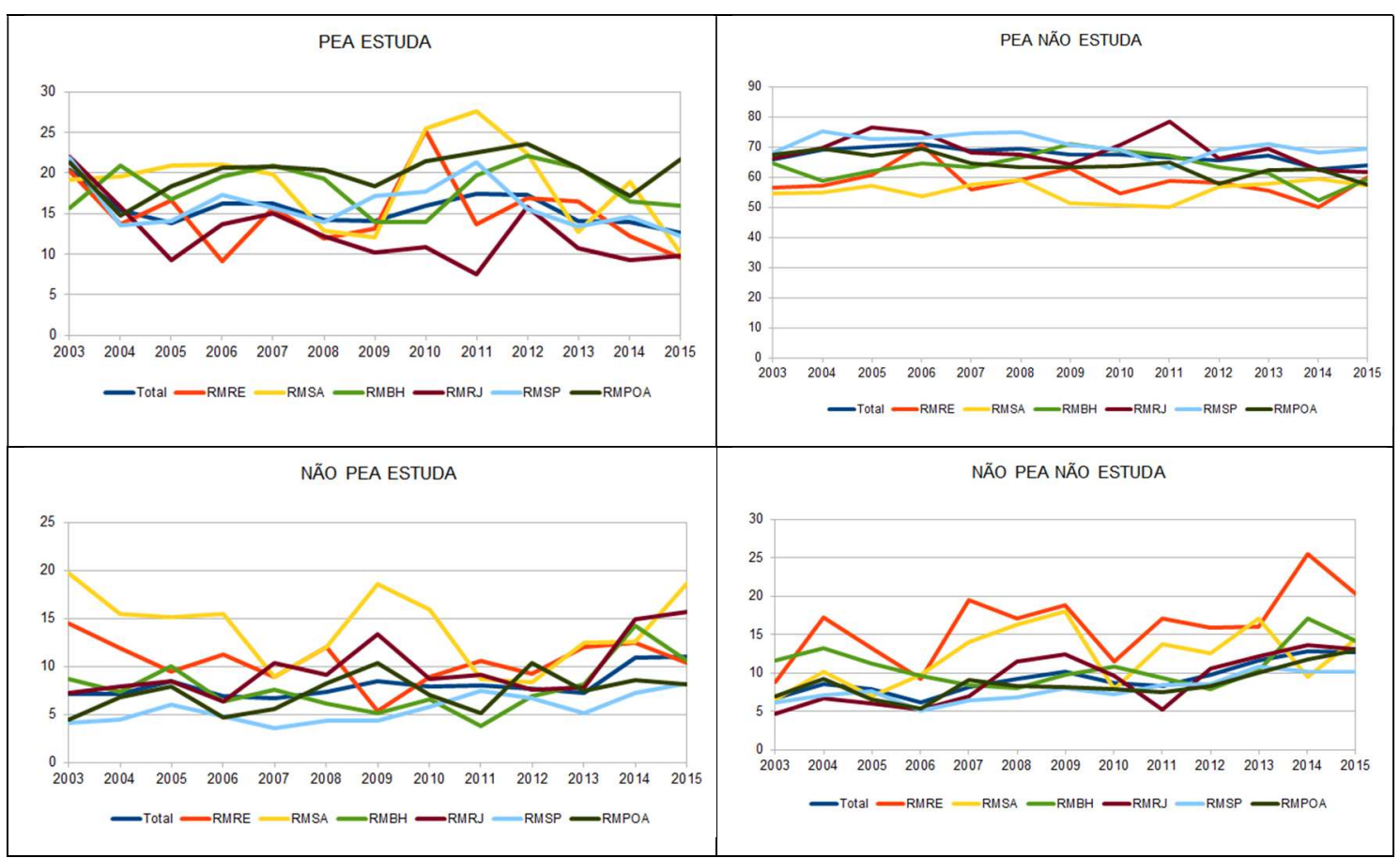

Fonte: Elaboração própria a partir dos microdados da PME

Para a análise dos resultados do modelo logit multinomial, utilizou-se como referência os jovens que estudam e participam da pea na quinta entrevista em relação aqueles que se encontram na pea e não estudam, que não se encontram na pea e estudam e que, ainda, não se encontram na pea e não estudam. As variáveis explicativas usadas são: condição na primeira entrevista, binárias de cor, binárias para o status domiciliar, sendo o chefe de domicílio a categoria de base, binárias de nível de estudo, tomando como referência jovens com menos de 3 anos de instrução formal; idade, em sua forma contínua, e, controles de período. Os resultados para as regiões metropolitanas de 2002 a 2015 são exibidos na Tabela 1 por meio da razão de chance, que indica maior ou menor probabilidade de se observar o evento em questão. 
p. 59 - Estudar, trabalhar ou alocar o tempo de outra maneira?

Tabela 1 - Razões de chance estimadas pelo modelo logit multinomial por região metropolitana, jovens do sexo masculino, 2002 a $2015^{2}$

\begin{tabular}{|c|c|c|c|c|c|c|c|}
\hline & & RMRE & RMSA & $\mathrm{RMBH}$ & RMRJ & RMSP & RMPOA \\
\hline \multicolumn{8}{|c|}{ PEA ESTUDA (REF) } \\
\hline \multicolumn{8}{|c|}{ NĀO PEA ESTUDA } \\
\hline & Não pea e estuda em 1 & 7,54 & 11,73 & 7,18 & 40,25 & 26,23 & 11,99 \\
\hline & Pea e não estuda em 1 & 2,42 & 3,18 & 1,85 & 3,62 & 2,64 & 2,16 \\
\hline & Não pea e não estuda em 1 & 11,69 & 7,51 & 7,10 & 27,05 & 14,32 & 6,81 \\
\hline & Brancos & 0,87 & 1,51 & 1,30 & 1,21 & 1,54 & 1,19 \\
\hline & Conjugue & $1, \infty$ & 0,57 & 0,78 & 0,33 & 1,78 & 0,00 \\
\hline & Filho & 1,10 & 1,32 & 1,18 & 1,25 & 1,54 & 2,24 \\
\hline & Outros & 0,93 & 1,23 & 1,75 & 1,66 & 1,44 & 2,83 \\
\hline & 4a 7 anos de estudo & 1,44 & 0,95 & 0,82 & 0,87 & 0,33 & 0,70 \\
\hline & 8 a 10 anos de estudo & 1,56 & 0,67 & 0,58 & 0,76 & 0,42 & 0,38 \\
\hline & 11 ou mais anos de estudo & 1,05 & 0,62 & 0,54 & $0, \pi 7$ & 0,31 & 0,44 \\
\hline & Idade & 0,88 & 0,88 & 0,83 & 0,90 & 0,91 & 0,92 \\
\hline & Constante & 2,80 & 3,82 & 16,67 & 0,63 & 0,60 & 0,52 \\
\hline \multicolumn{8}{|c|}{ PEA NĀO ESTUDA } \\
\hline & Não pea e estuda em 1 & 2,34 & 2,24 & 1,53 & 3,11 & 2,37 & 1,64 \\
\hline & Pea e não estuda em 1 & 10,20 & 9,06 & 9,42 & 24,82 & 14,13 & 9,93 \\
\hline & Não pea e não estuda em 1 & 21,44 & 10,57 & 11,60 & 20,77 & 17,14 & 8,74 \\
\hline & Brancos & 0,64 & 0,54 & 0,61 & 0,64 & 0,58 & 0,68 \\
\hline & Conjugue & 1,97 & 2,70 & 0,97 & 0,39 & 0,86 & 1,64 \\
\hline & Filho & 0,64 & 0,79 & 0,50 & 0,41 & 0,50 & 0,69 \\
\hline & Outros & 0,75 & 0,70 & 0,80 & 0,67 & 0,54 & 0,87 \\
\hline & 4 a 7 anos de estudo & 3,27 & 1,45 & 6,33 & 0,96 & 1,76 & 3,23 \\
\hline & 8 a 10 anos de estudo & 1,94 & 0,50 & 2,31 & 0,56 & 0,98 & 1,39 \\
\hline & 11 ou mais anos de estudo & 2,51 & 1,12 & 2,39 & 1,11 & 1,48 & 1,40 \\
\hline & Idade & 1,01 & 1,03 & 1,01 & 0,97 & 1,04 & 0,98 \\
\hline & Constante & 0,38 & 0,61 & 0,82 & 2,94 & 0,57 & 1,36 \\
\hline \multicolumn{8}{|c|}{ NĀO PEA NĀO ESTUDA } \\
\hline & Não pea e estuda em 1 & 7,22 & 7,20 & 4,70 & 27,10 & 12,02 & 6,26 \\
\hline & Pea e não estuda em 1 & 13,13 & 12,20 & 9,36 & 29,95 & 15,43 & 9,48 \\
\hline & Não pea e não estuda em 1 & 90,85 & 58,68 & 40,93 & 305,48 & 143,47 & 41,39 \\
\hline & Brancos & 0,62 & 0,78 & 0,58 & 0,58 & 0,64 & 0,69 \\
\hline & Conjugue & 1,85 & 2,45 & 1,25 & 1,26 & 1,16 & 0,27 \\
\hline & Filho & 1,96 & 1,88 & 1,79 & 1,75 & 1,60 & 1,60 \\
\hline & Outros & 2,22 & 1,47 & 2,46 & 2,82 & 1,47 & 1,67 \\
\hline & 4 a 7 anos de estudo & 1,87 & 0,64 & 2,31 & 0,32 & 0,44 & 0,64 \\
\hline & 8 a 10 anos de estudo & 0,56 & 0,12 & 0,55 & 0,11 & 0,15 & 0,22 \\
\hline & 11 ou mais anos de estudo & 0,90 & 0,32 & 0,43 & 0,34 & 0,18 & 0,18 \\
\hline & Idade & 0,97 & 0,98 & 0,93 & 0,91 & 0,92 & 0,97 \\
\hline & Constante & 0,10 & 0,18 & 0,74 & 0,41 & 0,92 & 0,48 \\
\hline
\end{tabular}

Fonte: Elaboração própria a partir dos microdados da PME

Os modelos estimados mostram uma significativa dependência temporal da condição anterior dos jovens, com destaque para aqueles que não faziam parte da pea e não estudavam e para aqueles que se encontram nessa condição no período atual. Como exemplo, a probabilidade de um jovem, na Região Metropolitana de Recife, que no primeiro ano não fazia parte da pea e estudava, um ano depois apresenta uma probabilidade 7,22 vezes maior de se encontrar na condição de nem-nem do que jovens que estudam e fazem parte da pea. Essa probabilidade aumenta para 13,13 para os jovens que faziam parte da pea, mas não estudavam na primeira entrevista e é ainda mais expressiva para aqueles que já se encontravam nessa condição. Isso pode ser associado à

${ }^{2}$ Nota: Coeficientes em negrito são não significativos a $10 \%$. 
uma maior vulnerabilidade dos jovens, pois na ausência do estudo, a dificuldade do retorno à educação pode ser dificultada por esta condição.

A dependência temporal significativa da posição em que o jovem se encontra na primeira entrevista, evidencia dificuldades para realizar transições principalmente para aqueles que se encontram na condição de nem-nem. Somando-se a isso, pelos controles individuais, os resultados mostram que jovens pretos e pardos, na condição de filhos, com menores níveis de escolaridade apresentam maior probabilidade de não fazerem parte da pea e não estudar. Por fim, fatores macroeconômicos, contribuem para esse comportamento no período mais recente.

\section{CONSIDERAÇÕES FINAIS}

Este artigo busca compreender como jovens de 18 a 24 anos do sexo masculino decidem alocar o tempo entre estudar, pertencer à pea ou combiná-la de outra maneira. Com base nos dados da PME, a análise abarca o período entre 2002 e 2015, quando a série é encerrada. Dois métodos de análise são empregados nas seis regiões metropolitanas abrangidas pela pesquisa, Recife, Salvador, Belo Horizonte, Rio de Janeiro, São Paulo e Porto Alegre. A influência de fatores individuais e relacionados à conjuntura sobre a decisão juvenil de alocação de seu tempo é feita por uma análise univariada por matrizes de transição e, uma multivariada, com o emprego de um modelo logit multinomial.

Os resultados apontam taxas de desocupação e de inatividade mais expressivas para jovens residentes nas regiões metropolitanas de Recife e Salvador enquanto Porto Alegre figura com as menores taxas. Diferenças ainda mais evidentes ao se considerar a escolaridade dos jovens, com destaque para o grupo de 8 a 10 anos de estudo, no qual a taxa de inatividade da região metropolitana do Rio de Janeiro figura também entre as mais altas. O emprego de matrizes de transição mostra um aumento na alocação do tempo em não estudar e não pertencer à população economicamente ativa (pea), com maior expressão para as regiões metropolitanas do Nordeste. Esse resultado pode ter impactos negativos na vida do jovem em um futuro próximo, comprometendo sua capacidade produtiva. Nessa condição, os jovens deixam de acumular capital humano em uma fase importante do ciclo de vida. A decisão por alocar tempo em estudar pode ser entendida como um investimento de um período mais longo do tempo, gerando resultados futuros sobre sua ocupação e sua renda.

Somando-se a isso, estimativas do modelo logit multinomial para cada região considerando a possibilidade de o jovem pertencer à pea e estudar, pertencer à pea e não estudar, não pertencer à pea e estudar e, ainda, não pertencer à pea e não estudar na quinta entrevista mostram dependência temporal significativa da posição em que o jovem se encontra na primeira entrevista, evidenciando dificuldades para realizar transições principalmente para aqueles que não se encontram na pea e não estudam. Jovens pretos e pardos, na condição de filhos, com menores níveis de escolaridade apresentam maior probabilidade de não fazerem parte da pea e não estudar. Fatores macroeconômicos contribuem para esse comportamento no período mais recente. Concluindo, o aumento da parcela juvenil masculina nem-nem e a expressiva dependência temporal dessa 
p. 61 - Estudar, trabalhar ou alocar o tempo de outra maneira?

condição apontam um cenário pouco favorável. Regionalmente, as regiões metropolitanas de Recife e Salvador são destaque. As maiores taxas de desocupação e de inatividade observadas para essas regiões contribuem para esse quadro, principalmente, para os jovens com ensino médio incompleto.

\section{REFERÊNCIAS}

BECKER, S. Gary. Human capital: a theoretical and empirical analysis, with special reference to education, 2nd ed. New York: Columbia University, 1975.

BEHRMAN, Jere; DE HOYOS, Rafael; SZÉKELY, Miguel. Out of school and out of work: a conceptual framework for investigating 'ninis' in Latin America and the Caribbean. World Bank. Washington, DC, 2014.

BORJAS, G. Economia do trabalho. McGraw-Hill. 5a ed. 2012.

CABANAS, P., KOMATSU, B. K, MENEZES-FILHO, N. O crescimento da renda dos adultos e as escolhas dos jovens entre estudo e trabalho. Insper Policy Paper n.13. 2015.

CAMARANO, A.A. (Org). Transição para a vida adulta oi vida adulta em transição? Rio de Janeiro: IPEA. 2006.

CLARK, Kim B.; SUMMERS, Lawrence H. Unemployment insurance and labor market transitions. In: SUMMERS, L. H. Understanding unemployment. Cambridge: MIT, 1990.

KOVROVA, I., LYON, S.. NEET youth dynamics in Indonesia and Brazil: a cohort analysis. Understanding Children's Work Programme Working Paper Series. 2013.

MENEZES-FILHO, N. A. M., CABANAS, P. H. F., KOMATSU, B. K. A condição "nem-nem" entre os jovens é permanente? Insper Policy Paper n.7. 2013.

SAMOILENKO, A., CARTER, K. Economic outcome of youth not in education, employment or training (NEET). New Zealand Treasury Working Paper 15/01. 2015.

SIMMONS, R. Raising the age of compulsory education in England: a NEET solution? British Journal of Educational Studies, 56(4), 420-439. 200

WOOLDRIDGE, J.M. Introdução à econometria: uma abordagem moderna. São Paulo: Thomson Learning, 2007. 


\section{ANEXO}

Tabela A - Razões de chance totais estimadas pelo modelo logit multinomial por região metropolitana, jovens do sexo masculino, 2002 a 201

\begin{tabular}{|c|c|c|c|c|c|c|c|c|c|c|c|c|c|}
\hline \multirow[t]{2}{*}{ PEAESTUDA (REF) } & & & & & & & & \multirow[b]{2}{*}{ RaARJ } & & \multirow[b]{2}{*}{ mansp } & & \multirow[b]{2}{*}{ RaPOA } & \\
\hline & & $\operatorname{mang}$ & & mansa & & ramen & & & & & & & \\
\hline NÃO PEA ESTUDA & & âde che & $\begin{array}{l}\text { Desvio- } \\
\text { apadtāo }\end{array}$ & axäo de char & $\begin{array}{l}\text { Desvio- } \\
\text { upactrāo }\end{array}$ & azäo de char & $\begin{array}{l}\text { Desvio- } \\
\text { Mpadtäo }\end{array}$ & azäo de char & $\begin{array}{l}\text { Desvio- } \\
\text { ppadtāo }\end{array}$ & azäo de char & $\begin{array}{l}\text { Desvio- } \\
\text { upadrāo }\end{array}$ & azäo de chan & $\begin{array}{c}\text { Desvio- } \\
\text { upaträo }\end{array}$ \\
\hline & Nẫo pea e estuda em 1 & 7,537 & 1,2684 & 11,725 & 1,8765 & 7,183 & B,8443 & 40,253 & 5,4152 & 26,27 & 4,0154 & 11,988 & 1,95066 \\
\hline & Pea e nẩo estuda em 1 & 2,424 & 8,4626 & 3,182 & B,5478 & 1,848 & 8,2488 & 3,617 & $\mathbf{8 , 5 7 6 4}$ & 2,635 & B,A310 & 2,156 & B,354 \\
\hline & Nǟ pea e nǟo estuda em 1 & 11,687 & 3,5577 & 7,514 & 2,1181 & 7,0988 & 1,4252 & 27,046 & 6,5165 & 14,323 & 3,7498 & 6,8056 & $1,0 / 41$ \\
\hline & Brancos & 0,869 & 8,1141 & 1,588 & 0,2093 & 1,295 & 8,1218 & 1,287 & 8,1166 & 1,537 & 8,2896 & 1,185 & 8,2592 \\
\hline & Conjugue & B,997 & 1,0344 & 0,574 & $0,6 / 37$ & 0,776 & 0,6426 & 0,332 & 0,2796 & 1,776 & 1,2452 & 0,000 & B,0080 \\
\hline & Filho & 1,098 & 0,4232 & 1,322 & 0,4025 & 1,178 & 0,3982 & 1,252 & 0,3264 & 1,540 & 0,4742 & 2,238 & 0,7102 \\
\hline & Outros & 0,931 & 0,4071 & 1,232 & 0,4216 & 1,754 & 0,6665 & 1,660 & 0,4919 & 1,437 & 0,5320 & 2,827 & 1,0838 \\
\hline & 4 a 7 anos de estudo & 1,436 & 0,5681 & 0,952 & 0,3896 & 0,819 & 0,3431 & 0,875 & 0,3105 & 0,329 & 0,1832 & 0,696 & $0,36 / 3$ \\
\hline & 8 a 10 anos de estudo & 1,556 & 0,5475 & 0,666 & 0,2550 & 0,575 & 0,1926 & 0,763 & 0,2586 & 0,417 & $0, \mathbf{1 9 2 0}$ & 0,379 & 0,1839 \\
\hline & 11 ou mais anos de estudo & 1,046 & 0,3540 & 0,620 & 0,2328 & 0,537 & 0,1763 & 0,768 & 0,2561 & 0,314 & 0,1469 & 0,444 & 0,2105 \\
\hline & Idade & 0,894 & 0,0362 & 0,883 & 0,0334 & 0,830 & 0,0239 & 0,995 & 0,0260 & 0,913 & 0,0380 & 0,920 & 0,0346 \\
\hline & 22804 & 0,878 & 0,2466 & 0,801 & 0,2357 & 0,608 & 0,1396 & 1,448 & 0,447 & 1,356 & 0,3949 & 1,490 & 0,4824 \\
\hline & 22005 & 0,932 & 0,2608 & $0, \pi / 8$ & 0,2237 & 0,837 & 0,1920 & 2,230 & 0,6586 & 1,762 & 0,4911 & 1,533 & 0,4881 \\
\hline & 22806 & 1,659 & 0,4832 & 0,794 & 0,2401 & 0,591 & $\mathbf{0 , 1 4 4 4}$ & 1,785 & 0,5217 & 1,330 & 0,3774 & 1,020 & 0,3391 \\
\hline & 22007 & 0,917 & 0,2695 & 0,726 & 0,2073 & 0,648 & 0,1571 & 1,924 & 0,5594 & 1,128 & $0,32 \pi 0$ & 1,148 & 0,3829 \\
\hline & a2008 & 1,241 & 0,3990 & 1,073 & 0,3399 & 0,553 & 0,1367 & 1,978 & 0,5783 & 1,439 & 0,4101 & 1,559 & 0,4973 \\
\hline & 22809 & 1,001 & 0,3585 & 1,357 & 0,4138 & 0,764 & B,1883 & 2,876 & 0,8606 & 1,160 & 0,3473 & 1,82 & 0,5838 \\
\hline & a2018 & 0,798 & 0,2658 & 0,820 & 0,2681 & 0,67 & 0,1735 & 2,873 & 0,6296 & 1,348 & D,3901 & 1,211 & 0,4638 \\
\hline & a2811 & 1,355 & 0,4722 & 0,423 & 8,1467 & 0,439 & 0,1128 & 2,489 & 0,7992 & 1,513 & 0,4401 & 1,072 & 0,3526 \\
\hline & a2012 & 0,872 & B,2818 & 0,585 & B,197 & 0,651 & 8,1573 & 1,449 & B,4433 & $1, m$ & B,5196 & 1,452 & B,4582 \\
\hline & a2013 & 1,146 & 8,3517 & 0,994 & B,3349 & 0,789 & B,1855 & 1,791 & 0,5458 & 1,637 & 0,5056 & 1,269 & B,4324 \\
\hline & a2014 & 1,249 & $0,36 \pi$ & B,, $\mathbf{B 1}$ & 8,2353 & 1,264 & B,2664 & 3,1058 & B,9483 & 2,266 & 0,5743 & 1,736 & B,5e98 \\
\hline & a2815 & 1,486 & B,43380 & 1,636 & B,5113 & 1,038 & B,22084 & 2,937 & 0,9228 & 2,601 & B,6834 & 1,457 & B,4346 \\
\hline & Constante & 2,891 & 3,0024 & 3,823 & 3,8099 & 16,63 & 13,2919 & 0,629 & B,5e89 & 0,603 & B,5598 & 0,519 & 0,5416 \\
\hline PEA NĀOESTUDA & & & & & & & & & & & & & \\
\hline & Nâ̄o pea e estuda em 1 & 2,337 & 0,3365 & 2,236 & 0,3129 & 1,535 & 0,1551 & 3,107 & 0,3487 & 2,373 & 0,2895 & 1,640 & 0,2193 \\
\hline & Pea e nāo estuda em 1 & 10,202 & 1,2988 & 9,056 & 1,0390 & 9,421 & $0, \pi 019$ & 24,817 & 2,2633 & 14,125 & 1,0842 & 9,925 & 0,9067 \\
\hline & Näo pea e nāo estuda em 1 & 21,443 & 5,6234 & $10,5 \pi 0$ & 2,3616 & 11,600 & 1,8465 & 20,768 & 4,3694 & 17,143 & 3,3413 & 8,740 & 1,5341 \\
\hline & Branoos & 0,635 & 0,0693 & 0,540 & 0,0748 & 0,605 & 0,0400 & 0,636 & 0,0495 & 0,581 & 0,0451 & 0,683 & 0,0938 \\
\hline & Conjugue & 1,975 & 1,2389 & $2, \pi 05$ & 1,4599 & 0,970 & 0,4233 & 0,388 & 0,1563 & 0,858 & $0,33 \pi$ & 1,637 & 0,7014 \\
\hline & Filho & 0,637 & 0,1732 & 0,787 & 0,1573 & 0,498 & 0,0883 & 0,410 & 0,0627 & 0,501 & 0,0736 & 0,685 & 0,0920 \\
\hline & Outros & 0,745 & 0,2323 & $0, \pi 1$ & 0,166 & 0,738 & 0,1663 & 0,673 & B,13e4 & 0,543 & 0,1080 & $0,8 \pi$ & 0,1760 \\
\hline & 4 a 7 anos de estudo & 3,266 & 1,1117 & 1,450 & B, 5079 & 6,333 & 2,1947 & 0,961 & 0,2754 & 1,758 & 0,7713 & 3,278 & 1,2541 \\
\hline & 8 a 18 anos de estudo & 1,943 & B,68B8 & 0,500 & $\mathbf{0 , 1 6 5 7}$ & 2,307 & 8,6872 & 0,562 & $\mathbf{0 , 1 5 3 9}$ & 0,975 & $\mathbf{0 , 3 9 4 9}$ & 1,3986 & B,5BAB \\
\hline & 11 ou mais anos de estudo & 2,514 & B,7396 & 1,116 & B,3595 & 2,392 & B, $\mathbf{B 1 4}$ & 1,106 & 0,2912 & 1,482 & B,5897 & 1,398 & B, 5801 \\
\hline & Idade & 1,007 & , 0339 & 1,029 & B,0383 & 1,006 & e,0199 & 0,907 & 0,0226 & 1,037 & 0,0218 & 0,981 & 0,0242 \\
\hline & a2804 & 1,240 & 0,2836 & 0,896 & 0,2151 & 0,663 & 0,1112 & 1,210 & 0,2961 & 1,302 & 0,2282 & 1,274 & 0,2632 \\
\hline & 22005 & 1,251 & 0,2947 & 0,899 & 0,2107 & 0,797 & $0, \mathbf{1 3 7 8}$ & 1,624 & 0,3839 & 1,243 & 0,2146 & 1,135 & 0,2287 \\
\hline & 22806 & 2,026 & 0,5066 & 0,907 & 0,2199 & 0,795 & 0,1390 & 1,304 & 0,3074 & 1,147 & 0,1962 & 1,118 & 0,2289 \\
\hline & 22807 & 1,303 & 0,3239 & 0,825 & 0,1946 & 0,749 & 0,1314 & 1,152 & 0,2697 & 1,189 & 0,2022 & 1,050 & 0,2159 \\
\hline & 22808 & 1,614 & 0,4317 & 1,348 & 0,3531 & 0,302 & 0,1402 & 1,131 & 0,2625 & 1,180 & 0,2038 & 0,996 & 0,2020 \\
\hline & a2009 & 1,428 & 0,4303 & 1,341 & 0,3504 & 0,980 & 0,1761 & 1,326 & 0,3228 & 1,047 & 0,1862 & 1,123 & 0,2317 \\
\hline & a2010 & 0,974 & 0,2639 & 0,776 & 0,1967 & 0,987 & 0,1774 & 1,387 & D,3441 & 0,964 & 0,1636 & 1,062 & 0,2288 \\
\hline & a2011 & 1,561 & 0,4733 & $0,6 / 9$ & 0,1759 & 0,792 & B,1395 & 1,541 & B,3924 & 0,852 & 0,1552 & 0,890 & 0,1846 \\
\hline & a2812 & 1,164 & B,380 & 0,757 & B,2179 & $8, \pi 08$ & 0,1224 & 0,985 & B,2393 & 1,139 & 0,2180 & 8,879 & B,1894 \\
\hline & 22013 & 1,005 & 0,2593 & 1,266 & B, 3826 & 0,719 & B,1266 & 1,210 & B,2942 & $1,2 \pi$ & 0,2453 & 0,955 & 0,2091 \\
\hline & 22814 & 1,271 & 0,3098 & 1,091 & 0,2663 & 0,719 & B,1194 & 1,339 & D,3296 & 1,155 & B,1950 & 1,108 & 0,2134 \\
\hline & 2015 & 1,600 & 0,4017 & 1,484 & 0,3883 & 0,784 & 0,1289 & 1,418 & 0,3619 & 1,250 & 0,2142 & 0,851 & 0,1626 \\
\hline & Constante & 0,378 & 0,3342 & 0,612 & 0,4986 & 0,816 & 0,4659 & 2,939 & 1,8565 & 0,569 & 0,3738 & 1,363 & 0,9655 \\
\hline NĀO PEA NĀOEST & & & & & & & & & & & & & \\
\hline & Nāo pea e estuda em1 & 7,223 & 1,6194 & 7,202 & 1,7429 & $4, \pi 04$ & 0,7624 & 27,697 & 6,0193 & 12,015 & 2,6392 & 6,263 & 1,4248 \\
\hline & Pea e não estuda em 1 & 13,127 & 2,7764 & 12,201 & 2,7122 & 9,357 & 1,2968 & 29,949 & 6,4700 & 15,425 & 2,8895 & 9,478 & 1,7386 \\
\hline & Näo pea e näo estuda em 1 & 90,854 & $28,05 \pi 0$ & 58,680 & 17,0016 & 40,926 & 8,0154 & 305,480 & 85,2525 & 143,472 & 36,6258 & 41,388 & 9,7463 \\
\hline & Brancos & $\mathbf{0 , 6 1 8}$ & B,B849 & 0,780 & 0,1537 & 0,5890 & B,0556 & 0,592 & 0,0623 & 8,640 & 0,0736 & 8,694 & 0,1271 \\
\hline & Conjugue & 1,855 & 1,6117 & 2,453 & 1,9226 & 1,253 & B,8481 & 1,260 & B,8368 & 1,164 & B,82899 & 0,273 & B,2343 \\
\hline & Filho & 1,962 & B, 7898 & 1, $\mathbf{9 8 9 0}$ & B,6462 & 1,795 & D,5123 & 1,52 & 0,5479 & 1,599 & 0,4478 & 1,596 & B,3957 \\
\hline & Outros & 2,223 & 8,9761 & 1,468 & 0,5728 & 2,464 & B,8281 & 2,816 & B,97/1 & 1,469 & B,5033 & $1,6 \mathrm{~B}$ & B,5436 \\
\hline & 4 a 7 anos de estudo & 1,866 & $0,66 / 8$ & 0,637 & 0,2291 & 2,312 & B,8014 & 0,318 & 0,0997 & 0,437 & 0,1894 & 0,643 & 0,2564 \\
\hline & 8 a 10 anos de estudo & 0,555 & 0,1842 & 0,121 & 0,0422 & 0,553 & 0,1634 & 0,114 & 0,0347 & 0,153 & 0,0603 & 0,220 & 0,0797 \\
\hline & 11 ou mais anos de estudo & 0,901 & 0,2753 & 0,320 & 0,1042 & 0,431 & 0,1242 & 0,343 & 0,0996 & 0,178 & $0,00 / 9$ & 0,180 & 0,0631 \\
\hline & Idade & 0,970 & 0,0393 & 0,983 & 0,0412 & 0,929 & 0,0257 & 0,909 & 0,0294 & 0,920 & 0,0302 & 0,972 & 0,0354 \\
\hline & a2004 & 1,419 & 0,4236 & 1,017 & 0,3612 & $0,7 N 0$ & 0,1760 & 1,293 & 0,4501 & 1,143 & 0,3187 & 1,424 & 0,4369 \\
\hline & 22805 & 1,374 & 0,4196 & 0,768 & 0,2824 & 0,894 & 0,2099 & 1,558 & 0,5257 & 1,363 & 0,3734 & 1,039 & 0,3180 \\
\hline & a2806 & 1,449 & 0,4753 & 1,022 & 0,3574 & 0,733 & 0,1848 & 1,215 & 0,4120 & 0,898 & 0,2547 & 0,668 & 0,2163 \\
\hline & a2807 & 1,994 & 0,6118 & 1,284 & 0,43999 & 0,639 & 0,1617 & 1,308 & D,4294 & 1,279 & 0,3471 & 1,126 & 0,3433 \\
\hline & a2088 & 2,064 & B,6854 & 2,059 & $0, \pi 27$ & B,618 & B,1554 & 1,841 & D,5966 & 1,344 & $0,37 n$ & 1,073 & D,3241 \\
\hline & 228009 & 1,824 & B,6612 & $2,5 \pi$ & B,9318 & 8,926 & B,2314 & 2,268 & B,7512 & 1,284 & B,3569 & 1,106 & B,3498 \\
\hline & a2810 & 1,029 & 0,3722 & 0,875 & B,3394 & 0,980 & B,2451 & 2,056 & B,6892 & 1,206 & B,3274 & 8,964 & B,3146 \\
\hline & a2811 & 1,963 & B,7488 & 0,996 & e,378s & 8,698 & 0,1737 & 1,357 & D,4859 & 1,200 & B,3488 & 0,977 & 0,3073 \\
\hline & a2812 & 1,636 & 0,5418 & 1,179 & 0,4869 & 0,547 & 0,1387 & 1,626 & 0,5529 & 1,418 & 0,4071 & 0,976 & 0,3023 \\
\hline & a2013 & 1,422 & 0,4765 & 2,499 & 1,0148 & 0,776 & 0,1926 & 2,341 & 0,7857 & 1,925 & 0,5651 & 1,111 & 0,3539 \\
\hline & 2014 & 2,737 & 0,8121 & 1,218 & 0,4350 & 1,323 & 0,2855 & $2, \pi / 8$ & 0,9278 & 1,874 & 0,4757 & 1,277 & 0,3633 \\
\hline & a2015 & 2,395 & 0,7316 & 2,205 & 0,8202 & 1,134 & 0,2448 & 2,913 & 1,0016 & 2,002 & 0,5157 & 1,360 & 0,3725 \\
\hline & Constante & 0,097 & 0,1059 & 0,177 & 0,1963 & 0,743 & 0,5698 & 0,406 & 0,3583 & 0,921 & 0,8518 & 0,479 & 0,4697 \\
\hline
\end{tabular}

